\title{
MicroRNAs and Lung Cancer: A Review Focused on Targeted Genes
}

\author{
Yao-Hui Wang ${ }^{1,2,3}$, Zhi-Ruo Zhu ${ }^{1,2,3}$, De Tong ${ }^{1,2,3}$, Rui Zhou ${ }^{1,2,3}$, \\ Kui Xiao ${ }^{1,2,3^{*}}$ and Ling Peng ${ }^{4 *}$
}

\begin{abstract}
${ }^{1}$ Department of Respiratory and Critical Care Medicine, The Second Xiangya Hospital of Central South University, Changsha, Hunan, China; ${ }^{2}$ Research Unit of Respiratory Disease, Central South University, Changsha, Hunan, China;

${ }^{3}$ The Respiratory Disease Diagnosis and Treatment Center of Hunan Province, Changsha, Hunan, China;

${ }^{4}$ Department of Respiratory Disease, Zhejiang Provincial People's Hospital, Hangzhou, Zhejiang, China
\end{abstract}

\begin{abstract}
Lung cancer is the leading cause of cancer morbidity and mortality. Surgery, chemotherapy and radiotherapy techniques have been developed over many years, and anti-angiogenic therapy, molecular targeted therapy and immune-checkpoint inhibitors have become increasingly effective for treating lung cancer. However, the overall disease-free and survival rates of lung cancer remain quite low. MicroRNAs are small, non-coding RNAs that consist of an average of 22 nucleotide molecules. MicroRNAs play an important role in the development, progression, metastasis, diagnosis and prognosis of lung cancer. This review summarizes the recent publications abnormally expressed miRNAs and the abnormal expression of their target genes in the biological process of lung cancer. This review aims to shed light on the recent advances in this field and to provide perspectives for future directions.
\end{abstract}

Introduction

Histologically, lung cancer can be divided into small cell lung cancer (SCLC) and non-small cell lung cancer (NSCLC). NSCLC is more common than SCLC, accounting for about $80 \%$ of all cases. NSCLC includes squamous cell carcinoma (SCC), adenocarcinoma, and large cell carcinoma, among which, adenocarcinoma and $\mathrm{SCC}$ are the most common subtypes. MicroRNAs (miRNAs) are small non-coding RNAs composed of an average of 22 nucleotides and are the most commonly studied non-coding RNAs in lung cancer. ${ }^{1}$ miRNAs mainly bind to the $3^{\prime}$ untranslated region of a target mRNA, which degrades target mRNA or blocks protein translation

Keywords: MicroRNAs; Lung cancer; Pathogenesis; Diagnosis; Therapy.

Abbreviations: SCLC, small cell lung cancer; NSCLC, non-small cell lung cancer; SCC, squamous cell carcinoma; miRNAs, microRNAs; LDHA, lactate dehydrogenase a; PDK4, pyruvate dehydrogenase kinase 4; EGFR, epidermal growth factor receptor; VEGF, vascular endothelial growth factor; TKI, tyrosine kinase inhibitor; PD-L1, programmed death-ligand 1.

Received: October 02, 2020; Revised: November 30, 2020; Accepted: December 11. 2020

*Correspondence to: Kui Xiao, Diagnosis and Treatment Center of Respiratory Disease, Research Unit of Respiratory Disease, Department of Respiratory and Critical Care Medicine, The Second Xiangya Hospital, Central South University, Changsha, Hunan, China. ORCID: http://orcid.org/0000-0001-6519-713X. Tel: +86-13973561934, E-mail: dr.kuixiao@csu.edu.cn; Ling Peng, Department of Respiratory Disease, Zhejiang Provincial People's Hospital, Hangzhou, Zhejiang, China. ORCID: http://orcid org/0000-0002-1359-4982. Tel: +86-571-85893509, Fax: +86-571-85893509, E-mail: drpengling@hotmail.com

How to cite this article: Wang Y-H, Zhu Z-R, Tong D, Zhou R, Xiao K, Peng L. MicroRNAs and Lung Cancer: A Review Focused on Targeted Genes. Exploratory Research and Hypothesis in Medicine 2020;000(000):000-000. doi: 10.14218/ERHM 2020.00058 . to regulate gene expression. miRNAs are involved in the regulation of the cell cycle, metastasis, angiogenesis, metabolism and apoptosis, and play an important role in the occurrence and development of tumors.

miRNAs in the development and progression of lung cancer

The occurrence and development of cancer is complex. The biological processes of cancer mainly include the occurrence of cancer, growth and metabolism, tumor microenvironment, neovascularization, tumor invasion and metastasis. A full understanding of the regulatory mechanisms and modes of action of miRNA in the occurrence and development of lung cancer may provide new strategies for the diagnosis and treatment of lung cancer. In the following sections, we will focus on the role of miRNAs in these processes.

\section{miRNAs in the occurrence of lung cancer}

The role of miRNAs in the occurrence of lung cancer manifests as targeting both oncogenes and tumor suppressor genes. The causes of lung cancer are complex and are mainly related to abnormal genes. These abnormalities usually occur in areas where genes are not stable. Studies have found that a transcriptional hyper-conserved region gene is located in an unstable genomic region associated with cancer. This super-conserved region consists of a genomic sequence family of more than 200 base pairs (bp) in length. Most transcriptional hyper-conserved region 
Table 1. MicroRNAs involved in the cell cycle and apoptosis

\begin{tabular}{|c|c|c|c|c|}
\hline miRNAs & Targets/pathway & Tumor suppressor/oncogene & Lung cancer type & Ref \\
\hline $\operatorname{miR} 485-5 p$ & IGF2BP2 & suppressor & NSCLC & Huang et al. ${ }^{17}$ \\
\hline $\operatorname{miR} 183$ & MTA1 & suppressor & NSCLC & Yang et al. ${ }^{18}$ \\
\hline miR335 & $\operatorname{Tra} 2 \beta$ & suppressor & NSCLC & Liu et al. ${ }^{19}$ \\
\hline $\operatorname{miR} 188$ & МАР3К3 & suppressor & NSCLC & Zhao et al. ${ }^{20}$ \\
\hline $\operatorname{miR} 186$ & SIRT6 & suppressor & NSCLC & Ruan et al. ${ }^{21}$ \\
\hline miR93-5p & PTEN and RB1 & oncogene & NSCLC & Yang et al. ${ }^{22}$ \\
\hline miR4326 & APC2 & oncogene & NSCLC/SCLC & Xu et al. ${ }^{23}$ \\
\hline miR339 & Skp2 & suppressor & NSCLC/SCLC & Ren et al. ${ }^{24}$ \\
\hline miR520b & HDAC4 & suppressor & NSCLC/SCLC & Jin et $a .^{25}$ \\
\hline $\operatorname{miR628-3p}$ & HSP90 & suppressor & NSCLC/SCLC & Pan et $a .^{26}$ \\
\hline let7a & cyclin D1 & suppressor & Human Lung adenocarcinoma & Zhao et al. ${ }^{27}$ \\
\hline miR505 & MAP3К3 & suppressor & NSCLC & Tang et $a .^{28}$ \\
\hline $\operatorname{miR} 135 a$ & IGF1 & suppressor & NSCLC & Zhou et al. ${ }^{16}$ \\
\hline
\end{tabular}

genes are not translated into proteins and act as oncogenes to promote carcinogenesis by inhibiting miRNAs. ${ }^{2}$ There are also some miRNAs involved in the development of lung cancer. Oncogenic miRNA-411 promotes lung cancer by directly targeting the inhibitory genes SPRY4 and TXNIP. ${ }^{3}$ MiR-30c and miR-21 are significantly up-regulated by the oncogene KRAS isotype, induces drug resistance and enhances cell migration or invasion by inhibiting key tumor suppressor genes such as NF1, RASA1, BID and RASSF $8 .{ }^{4}$ In lung cancer cell lines, the up-regulation of miR-365a-3p targets and down-regulates USP33, and promotes the proliferation, migration, and invasion of lung cancer cells via the miR-365/USP33/SLIT2/ROBO1 axis. ${ }^{5}$ Another study found that the up-regulation of the novel miR-12528 directly targets the $3^{\prime}$ untranslated region of the insulin-like growth factor 1 receptor (IGF-1R) mRNA, negatively regulates proliferation, apoptosis and migration activity through hypophosphorylation of the AKT cascade, and thus inhibits tumorigenesis and metastasis in vivo. ${ }^{6}$ In addition to genetic abnormalities, external factors such as smoking also play an important role in the development of lung cancer. In fact, miRNAs have been found to be involved in the regulation of smoking-related oncogenes. ${ }^{7}$ Continuous smoking induces the development of non-small cell lung cancer by reducing the expression of microRNA, including hsa-mir-185-3p, hsamir-4295, hsa-mir-4288, and hsa-mir-613, associated with the ERBB pathway. ${ }^{8}$ Nicotine promotes NSCLC cell proliferation and epithelialtomesen-chymaltransition by down-regulating miR$99 \mathrm{~b}$ and miR-192. ${ }^{9}$ In addition, miR21, which is involved in the regulation of HIF $1 \alpha$, plays a role in the malignant transformation of HBE cells which is induced by cigarette smoke extract through the Akt/NF- $\kappa \mathrm{B}$ pathway. ${ }^{10}$ Simultaneous radiation exposure can enhance epithelial-mesenchymal transition (EMT) of A549 cells via miR3591-5p/USP33/PPM1A. ${ }^{11}$

\section{miRNAs involved in tumor growth and metabolism}

miRNAs involved in the cell cycle and apoptosis

The cell cycle is the basis of cell proliferation, which, in addition to apoptosis, are two important processes of tumor growth and are closely related to miRNAs. Of note, miR21 inhibitors promote apoptosis by inhibiting the $\mathrm{PI} 3 \mathrm{~K} / \mathrm{Akt} / \mathrm{NF}-\mathrm{\kappa B}$ signaling pathway in NSCLCs in vitro and in vivo. ${ }^{12}$ Also, miR19b enhances proliferation and apoptosis resistance through the epidermal growth factor receptor (EGFR) signaling pathway in NSCLC by targeting PP2A and BIM. ${ }^{13}$ Overexpression of miR143 significantly reduces cell proliferation and promotes apoptosis, ${ }^{14}$ and miR335-5p inhibits cell proliferation by targeting CPNE1 in NSCLC. ${ }^{15}$ Other miRNAs involved in cell cycle and apoptosis are shown in Table 1.16-28

miRNAs involved in the metabolism of lung cancer

Altered metabolism is an important feature in the development of cancer. Various miRNAs can directly or indirectly participate in various metabolic processes of lung cancer cells, including glucose metabolism, amino acid metabolism and lipid metabolism, ${ }^{29}$ which provide the rapidly multiplying cells with much needed energy. At present, the research on the metabolism of miRNAs in lung cancer is mostly studied by glucose metabolism. High glucose promotes cell proliferation, migration and invasion in NSCLCs. ${ }^{15}$ By reviewing articles on miRNAs and lung cancer metabolism, it has been found that miRNAs involved in cancer metabolism mainly play a role in the regulation of a series of biological enzymes involved in the metabolic processes..$^{30}$ The Warburg effect (aerobic glycolysis) is a common feature of cancer cells, which facilitates tumor cell proliferation and progression with elevated glucose uptake and lactate production. ${ }^{31}$ Lactate dehydrogenase A (LDHA), one of the subunits of lactate dehydrogenase, participates in the final step of the aerobic glycolysis process by catalyzing pyruvate into lactate. In fact, a recent study found that miR200c can inhibit the proliferation and migration of NSCLC cells by down-regulating LDHA. ${ }^{32}$ Downregulation of miR33b promotes NSCLC cell growth by reprogramming glucose metabolism. ${ }^{33}$ In NSCLC cells, down-regulated miR214 inhibits cell proliferation and glycolysis by reducing the expression of HK2 and PKM2 via the PTEN/Akt/mTOR pathway. ${ }^{34}$ Pyruvate dehydrogenase kinase 4 (PDK4) and pyruvate dehydrogenase (PDH) are important biological enzymes in sugar metabolism and fatty acid synthesis, respectively. It has been reported 
Table 2. MicroRNAs that affect tumorigenesis through the tumor microenvironment

\begin{tabular}{lllll}
\hline miRNA & target & Tumor suppressor/oncogene & Lung cancer type & Ref \\
\hline miR105 & Mcl1 & oncogene & NSCLC & Jin et al. ${ }^{42}$ \\
miR505 & MAP3K3 & suppressor & NSCLC & Tang et al. ${ }^{28}$ \\
miR3127-5p & FZD4 & suppressor & NSCLC & Yang et al. ${ }^{43}$ \\
miR182 & Met & suppressor & NSCLC & Li et al. ${ }^{44}$ \\
miR598 & DERL1 & suppressor & NSCLC & Yang et al. ${ }^{45}$ \\
miR145/miR497 & MTDH & suppressor & NSCLC & Yin et al. ${ }^{46}$ \\
miR103a & PTEN & oncogene & NSCLC/SCLC & Hsu et al. ${ }^{47}$ \\
miR1246 & CXCR4 & suppressor & NSCLC/SCLC & Xu et al. ${ }^{48}$ \\
miR138 & SOX4/p53 & suppressor & NSCLC & Li et al. ${ }^{49}$ \\
\hline
\end{tabular}

that the overexpression of miR-182 can regulate PDH through the miR182-PDK4 axis. PDK4 is activated and lipogenesis promotes lung cancer cell proliferation and tumor growth. Overexpression of miR182 and PDK4 knockdown significantly promotes triglyceride levels, suggesting that miR182 and PDK4 affect lipogenesis in lung cancer cells. ${ }^{29}$ Another miRNA, miR198, inhibits the proliferation of lung adenocarcinoma cells in vitro and in vivo by directly targeting SHMT1, which leads to enhanced apoptosis and leads to cell cycle arrest in lung adenocarcinoma. ${ }^{35}$

\section{miRNAs involved in the formation of the tumor microenviron- ment and angiogenesis}

miRNAs involved in the tumor microenvironment of lung cancer

Disordered miRNAs can affect cancer proliferation, angiogenesis, tumor metastasis, etc. by regulating the tumor microenvironment. ${ }^{36}$

miRNAs involved in tumor angiogenesis

Neovascularization ensures that the required nutrients and oxygen are brought to the tumor tissue and is a backup force in the progression of the tumor. Many studies have demonstrated that miRNAs have a regulatory role in tumor angiogenesis. ${ }^{37}$ Vascular endothelial growth factor (VEGF) is more important in this process because it directly stimulates vascular endothelial cells to promote neovascularization and increase microvascular permeability. It has also been found that miR143/145 in lung adenocarcinoma significantly promotes tumor angiogenesis by stimulating the proliferation of endothelial cells, mainly through the targeting of Camk1d (an inhibitory kinase) by miR145. ${ }^{38}$ There is also a lung cancerderived exosome miR23a that increases angiogenesis and vascular permeability by targeting prolyl hydroxylase and tight junction protein ZO-1 under hypoxic conditions. ${ }^{39}$ Studies have shown that in NSCLC, miR1 can reduce VEGF-induced mouse lung endothelial cell proliferation. The miRNA also reduces de novo DNA synthesis by targeting the thrombopoietin receptor in lung endothelial cells and by activating extracellular signal-regulated protein kinase $1 / 2$ in human umbilical vein endothelial cells to inhibit tumor growth and angiogenesis. ${ }^{40}$ MiR135a inhibits tumor angiogenesis by inhibiting IGF1 by decreasing the expression of angiogenesisrelated factors VEGF, bFGF and IL8 in A549 cells. ${ }^{16}$ Furthermore, miR204 may attenuate angiogenesis in lung adenocarcinoma via the JAK2-STAT3 pathway. ${ }^{41}$ These studies have shown that miR-
NAs play various roles in tumor angiogenesis. Other miRNAs that affect tumorigenesis through the tumor microenvironment are shown in Table 2.28,42-49

\section{miRNAs involved in tumor invasion and metastasis}

Invasion and metastasis are complicated processed that are both important causes of the poor prognosis of cancer. A large number of studies have found that miRNA expression profiles are closely related to the invasion and metastasis of lung cancer. Exosomal-mediated miR193a-3p, miR210-3p and miR5100 metastasis can promote lung cancer cell invasion by activating STAT3 signaling-induced EMT. ${ }^{50}$ Overexpressed miR302b-3p inhibits the proliferation, migration and invasion of NSCLC cells by the direct targeting of GCNT3.5 In NSCLC, miR409 inhibits the growth, proliferation and migration of cancer cells by directly targeting SPIN $1 .^{52}$ MiR-520a3p inhibits proliferation, migration and invasion of NSCLC via the PI3K/AKT/ mTOR signaling pathway. ${ }^{53}$ A summary of the miRNAs involved in tumor invasion and metastasis are shown in Table 3.22,54-79

\section{The role of miRNAs in the diagnosis of lung cancer}

The lack of effective means of early diagnosis is the main cause of the high mortality rate of lung cancer. As such, miRNAs are often dysregulated in lung cancer and form a specific expression spectrum, which is conducive to the diagnosis of lung cancer. In a metaanalysis, the authors claimed that they could determine if a person has lung cancer based on whether 11 miRNAs, including miR210, miR21, miR155, were contained in a sputum specimen $(1,009$ NSCLC patients and 1,006 controls).$^{80}$ It has also been found that the combination of miR205-5p and miR210-3p may be useful in the diagnosis of early stage lung cancer. ${ }^{81}$ Relevant studies found that by analyzing the serum of lung cancer patients that the expression of miR661, miR441 and miR181B-5 was significantly increased compared with healthy controls. These results indicated that miRNAs can be used as serum markers for the diagnosis of lung cancer. Overall, the efficacy of a combined imaging approach for the early diagnosis of lung cancer was significantly increased. ${ }^{82,83}$

The role of miRNAs in the treatment of lung cancer

Chemotherapy, radiotherapy, targeted therapy and ICI therapy are 
Table 3. MicroRNAs involved in tumor invasion and metastasis

\begin{tabular}{|c|c|c|c|c|}
\hline miRNA & targets & Tumor suppressor/oncogene & Lung cancer type & Ref \\
\hline $\operatorname{miR} 210$ & LOXL4 & oncogene & lung adenocarcinoma & Xie et al..$^{54}$ \\
\hline $\operatorname{miR} 342-3 p$ & AGR2 & suppressor & NSCLC & Xue et al. ${ }^{55}$ \\
\hline $\operatorname{miR3666}$ & BPTF & suppressor & Lung adenocarcinoma & Pan et al..$^{56}$ \\
\hline $\operatorname{miR} 146-5 p$ & claudin12 & oncogene & NSCLC/SCLC & Sun et al. ${ }^{57}$ \\
\hline miR33a & CAND1 & suppressor & NSCLC/SCLC & Kang et al. ${ }^{58}$ \\
\hline miR889 & KLF9 & oncogene & NSCLC & Han et al. ${ }^{59}$ \\
\hline $\operatorname{miR93-5p}$ & PTEN and RB1 & oncogene & NSCLC & Yang et al. ${ }^{22}$ \\
\hline miR103 & PDCD10 & suppressor & NSCLC & Yang et al. ${ }^{60}$ \\
\hline $\operatorname{miR223-5p~}$ & E2F8 & suppressor & NSCLC & Dou et al. ${ }^{61}$ \\
\hline miR449a & HMGB1 & suppressor & NSCLC & Wu et $a .^{62}$ \\
\hline miR373 & BRF2 & suppressor & NSCLC & Wang et al. ${ }^{63}$ \\
\hline $\operatorname{miR} 214$ & JAK1 & suppressor & NSCLC/SCLC & Chen et al. ${ }^{64}$ \\
\hline miR101 & ZEB1 & suppressor & NSCLC & Han et al. ${ }^{65}$ \\
\hline miR204 & PCNA1 & suppressor & NSCLC/SCLC & Li et al. ${ }^{66}$ \\
\hline $\operatorname{miR758}$ & HMGB3 & suppressor & NSCLC & Zhou et al. ${ }^{67}$ \\
\hline miR497-5p & SOX5 & suppressor & NSCLC & Li et al. ${ }^{68}$ \\
\hline $\operatorname{miR} 1246$ & GSK3 $\beta$ & oncogene & NSCLC & Yang et al. ${ }^{69}$ \\
\hline $\operatorname{miR} 362$ & Sema3A & oncogene & NSCLC & Luo et al. ${ }^{70}$ \\
\hline $\operatorname{miR} 128-3 p$ & Drosha and Dicer & oncogene & NSCLC & Frixa et al. ${ }^{71}$ \\
\hline miR320a-3p & PI3K/Akt pathway & suppressor & NSCLC & Zhao et $a .^{72}$ \\
\hline $\operatorname{miR} 875-5 p$ & SATB2 & oncogene & NSCLC & Wang et al. ${ }^{73}$ \\
\hline miR26a-5p & ITG $\beta 8$ & oncogene & NSCLC, SCLC & Song et $a l^{74}$ \\
\hline miR320a & $\mathrm{p} 100$ & suppressor & NSCLC, SCLC & Xing et $a .^{75}$ \\
\hline miR145-3p & PDK1 & suppressor & NSCLC & Chen et al. ${ }^{76}$ \\
\hline $\operatorname{miR} 212$ & USP9X & suppressor & NSCLC & Chen et al. ${ }^{77}$ \\
\hline miR24-3p & sox7 & oncogene & NSCLC, SCLC & Yan et al. ${ }^{78}$ \\
\hline $\operatorname{miR} 150$ & SIRT2/JMJD2A pathway & oncogene & NSCLC & Jiang et $a .^{79}$ \\
\hline
\end{tabular}

widely used for lung cancer treatment and different miRNAs are involved in the sensitivity and drug resistance thereof.

\section{Chemotherapy}

Platinum drugs, such as cisplatin and carboplatin, are commonly used in lung cancer chemotherapy regimens; however, the resistance of NSCLC cells to platinum-based drugs is a common cause of poor efficacy. Therefore, miRNAs are associated with chemotherapy sensitivity and drug resistance. The sensitivity of cisplatin in miR155 overexpressing NSCLC cell lines is reduced. ${ }^{84}$ Furthermore, in NSCLC, the up-regulation of miR128-3p may overactivate $\mathrm{Wnt} / \beta$-catenin and TGF $\beta$ signaling and confer resistance to chemotherapy-resistant metastasis. ${ }^{85}$ Some studies have also found that miR96-reduced cisplatin-induced NSCLC cell apoptosis is caused by down-regulating SAMD9 expression. ${ }^{86}$ Other miRNAs that are associated with chemosensitivity or resistance to lung cancer are shown in Table $4 .^{\mathbf{8 5 , 8 7 - 9 7}}$

\section{Radiotherapy}

Radiotherapy is a treatment that uses radioactive rays to destroy cancer cells and is suitable for selected NSCLC and SCLC patients. However, the efficacy of radiotherapy is limited and some patients are prone to relapse, which may be due to radiation resistance in their cancer cells. Studies have shown that cell radiosensitivity is associated with apoptosis as well as cell cycle and DNA damage. Therefore, it is particularly important to find the cause and markers that affect radiotherapy resistance. Studies have shown that, in vitro, miR155 reduces the radiotherapy sensitivity of lung cancer by inhibiting FOXO3A and TP53INP1. By contrast, inhibiting the expression of miR155 can improve the radiotherapy effect. ${ }^{98}$ Shin et al..$^{99}$ studied the human lung adenocarcinoma cell line A549 and found that the expression level 
Table 4. MicroRNAs associated with chemosensitivity or resistance to lung cancer

\begin{tabular}{|c|c|c|c|c|}
\hline miRNA & Up/down & Target/pathway & Medicine & Ref. \\
\hline \multicolumn{5}{|c|}{ Chemo-sensitive } \\
\hline miR9 & UP & elF5A2 & Cisplatin & Cai et al. ${ }^{85}$ \\
\hline miR539 & UP & DCLK1 & cisplatin & Deng et $a .^{88}$ \\
\hline miR202 & UP & Ras/MAPK Pathway & cisplatin & Sun et $a .^{90}$ \\
\hline miR155 & UP & miR155/TP53 feedback loop & Cisplatin & Van Roosbroeck et al. ${ }^{92}$ \\
\hline miR106b-5p & UP & PKD2 & Cisplatin & Yu et al. ${ }^{94}$ \\
\hline $\operatorname{miR} 140$ & UP & SIRT1/ROS/JNK & Cisplatin & Lin et al. ${ }^{96}$ \\
\hline \multicolumn{5}{|c|}{ Chemo-resistant } \\
\hline miR130b & UP & PTEN & cisplatin & Zhang et al. 87 \\
\hline miR181b & Down & $\mathrm{Bcl} 2$ & cisplatin & Liu et al. ${ }^{89}$ \\
\hline $\operatorname{miR221}$ & UP & PTEN & cisplatin & Wang et al. ${ }^{91}$ \\
\hline miR133b & Down & GSTP1 & cisplatin & Lin et $a ._{.93}$ \\
\hline $\operatorname{miR} 144-3 p$ & UP & Nrf2 & cisplatin & Yin et al. ${ }^{95}$ \\
\hline miR324-5p & UP & FBX011 & cisplatin & Ba et al. ${ }^{97}$ \\
\hline
\end{tabular}

of 8 miRNAs changed after 20 Gy and 40 Gy-exposure, while that of 10 miRNAs changed only after 40 Gy-exposure. Studies have found that the down-regulation of miR18a expression can increase the sensitivity of NSCLC cells to radiotherapy. ${ }^{100}$ Other miRNAs that associated with sensitivity or resistance to lung cancer radiotherapy are shown in Table $5 .{ }^{101-108}$

\section{Molecular targeted therapy}

Many studies have reported the role of various miRNA expressions in targeted therapies. Tyrosine kinase inhibitor (TKI) is a small molecule that targets the intracellular tyrosine signaling pathway. EGFR is a glycoprotein receptor consisting of 1,186 amino acid residues with a molecular weight of $170 \mathrm{kD}$. After activation, EGFR can lead to intracellular tyrosine kinase activation and phosphorylation through copolymerization to activate downstream RAS-Raf-MAPK, PI3K-Akt, and JAK/STAT pathways. This process can thereby mediate tumor cell proliferation, angiogenesis, and apoptosis inhibition. Interestingly, TKIs such as gefitinib and erlotinib are often used to treat EGFR-sensitive mutant lung cancer patients. The miRNA, miR483-3p, reverses EMT and inhibits migration, invasion and metastasis of gefitinibresistant lung cancer cells. ${ }^{109}$ This indicates that the overexpression of Mir483-3p can effectively improve the sensitivity of gafitinib-resistant lung cancer cells to gafitinib. Other miRNAs that are associated with molecular targeted therapy for lung cancer are shown in Table $6 .{ }^{109-114}$

\section{Immunotherapy}

There are programmed death-1 (PD-1) proteins in the membrane of T-cells, which, if bound with the programmed death-ligand 1 (PD-L1) on tumor cells, can be redirected to kill these tumor cells. While this theory exists, little research has been published in this field. The up-regulation of miR140 in NSCLC directly inhibits the PD-L1 and the PD-L1/cyclin E pathway to inhibit cell pro-

Table 5. MicroRNAs associated with sensitivity or resistance to lung cancer radiotherapy

\begin{tabular}{|c|c|c|c|}
\hline miRNA & Up/down & Target/pathway & Ref. \\
\hline \multicolumn{4}{|c|}{ Radio-sensitive } \\
\hline $\operatorname{miR373}$ & Up & TIMP2 & Guo et al. ${ }^{101}$ \\
\hline miR18a-5p & Up & ATM and HIF1 $\alpha$ & Chen et al. ${ }^{103}$ \\
\hline miR200a & Up & HGF/c-Met pathway & Jiang and Du et al. ${ }^{104,105}$ \\
\hline miR144-5p & Up & ATF2 & Song et al. ${ }^{107}$ \\
\hline miR99a & Up & $\begin{array}{l}\text { ATF2 } \\
\text { mTOR }\end{array}$ & Yin et al. ${ }^{108}$ \\
\hline \multicolumn{4}{|c|}{ Radio-resistant } \\
\hline miR198 & down & HGF/c-MET pathway & Zhu et al. ${ }^{102}$ \\
\hline $\operatorname{miR} 21$ & UP & HIF1 $\alpha$ & Jiang et al..$^{104}$ \\
\hline $\operatorname{miR} 1323$ & down & PRKDC & Li et al. ${ }^{106}$ \\
\hline
\end{tabular}


Table 6. MicroRNAs associated with molecular targeted therapy for lung cancer

\begin{tabular}{|c|c|c|c|c|}
\hline miRNA & Up/down & Target/pathway & Medicine & Ref. \\
\hline \multicolumn{5}{|c|}{ Target- sensitive } \\
\hline miR135a & UP & RAC1 & Gefitinib & Zhang et al. ${ }^{110}$ \\
\hline miR200c & UP & PI3K/Akt pathway & Gefitinib & Zhou et al. ${ }^{112}$ \\
\hline \multicolumn{5}{|c|}{ Target-resistant } \\
\hline miR181a & UP & GAS7 & Gefitinib & Ping et al. ${ }^{111}$ \\
\hline $\operatorname{miR} 873$ & UP & GLI1 & Gefitinib & Jin et $a .^{113}$ \\
\hline $\operatorname{miR} 138$ & Down & HOXA4 & Gefitinib & Tang et al. ${ }^{114}$ \\
\hline $\operatorname{miR} 483-3 p$ & UP & integrin $\beta 3 / F A K / E r k$ pathway & Gefitinib & Yue et al. ${ }^{109}$ \\
\hline
\end{tabular}

liferation. These results suggest that the miR140/PD-L1/cyclin E pathway may be a potential therapeutic target for the inhibition of NSCLC cell proliferation. ${ }^{115}$

\section{The role of miRNAs in the prognosis of lung cancer}

In a study of the prognostic role of circulating miRNAs in early NSCLC, five miRNAs (miR26a-5p, miR126-3p, miR130b-3p, miR205-5p and miR21-5p) were found to be significantly associated with disease-free survival (DFS) in SCC after surgical operation. Furthermore, four miRNAs (miR130b-3p, miR26a$5 p$, miR $126-3 p$ and miR205-5p) were found to be significantly correlated with overall survival. In adenocarcinoma, miR222$3 p$, miR22-3p and miR93-5p were significantly associated with DFS, ${ }^{116}$ and other miRNAs are thought to be involved in the prognosis of lung cancer. The 5-year survival rate of patients with low expression of miR455-3p in SCLC is significantly shorter than that of patients with high expression of miR455-3p. ${ }^{117}$ High expression of miR421 is associated with positive lymph node metastasis and advanced TNM staging, and has been shown to be an independent prognostic factor for NSCLC. ${ }^{118}$ Serum miR150 predicts the prognosis of early (stage I-II) NSCLC and can promote tumor cell proliferation by targeting the tumor suppressor gene SRCIN1.119

\section{Future directions}

Studies have shown that the abnormal expression of miRNAs can be detected in many types of tumors and that miRNAs play an important role in tumorigenesis. A large number of studies have reported on the relationship between miRNAs and lung cancer and have shown that miRNAs may bring certain benefits to the early diagnosis, treatment and prognosis of lung cancer. In fact, miRNAs may be a target for drug development, and can directly participate in the proliferation, apoptosis, metabolism, invasion and metastasis of lung cancer cells or indirectly enhance the sensitivity of chemotherapy, radiotherapy, targeted therapy or immunotherapy. However, due to the large number of miRNAs and the complexity and diverse regulatory pathways of lung cancers, there are still many questions to be answered.

As of now, experts have extensively investigated the relationship between microRNAs and lung cancer, but are still looking for miRNAs that can be stably expressed in the human body and that can be helpful in the diagnosis and treatment of tumors. We expect more work to participate in studying the relationship between mi-
croRNAs and lung cancer, which is expected to bring benefits to human health in the future.

\section{Conclusions}

Although there are a large number of studies on the relationship between miRNAs and lung cancer, miRNAs have not been applied to the clinical diagnosis and treatment of lung cancer. There may be a number of issues that need to be considered before a miRNA can be used in a clinical setting. For example, the safety of miRNA in the application process must be evaluated, in addition to whether a miRNA has high sensitivity and specificity for different individuals. Furthermore, it must be examined whether a miRNA is suitable for all patients and can be continuously and stably expressed in vivo. Nevertheless, the role of miRNAs in lung cancer research has attracted many attentions and will continue to provide insights into this disease as more mechanisms are revealed.

\section{Acknowledgments}

None.

Funding

This work was supported by the Excellent-Surpass-Climb Disciplines Project of The Second Xiangya Hospital of Central South University; the Scientific Research Project of Hunan Provincial Health Commission, (No. 202103020704) and the National Key Clinical Specialty Construction Projects of China.

\section{Conflict of interest}

The authors declare that there is no conflict of interest.

\section{Author contributions}

Conceptualization (YHW, DT), methodology (DT, ZRZ), writing of the original draft (YHW, DT, KX), writing, review and editing of the manuscript (YHW, KX, LP, ZRZ). All authors have made an intellectual contribution to the manuscript and approved the submission. 
References

[1] Braicu C, Zimta AA, Harangus A, lurca I, Irimie A, Coza O, et al. The Function of Non-Coding RNAs in Lung Cancer Tumorigenesis. Cancers (Basel) 2019;11(5):605. doi:10.3390/cancers11050605.

[2] Zhen Q, Gao LN, Wang RF, Chu WW, Zhang YX, Zhao XJ, et al. LncRNA DANCR Promotes Lung Cancer by Sequestering miR-216a. Cancer Control 2018;25(1):1073274818769849. doi:10.1177/1073274 818769849.

[3] Zhang C, Wang H, Liu X, Hu Y, Ding L, Zhang X, et al. Oncogenic microRNA-411 promotes lung carcinogenesis by directly targeting suppressor genes SPRY4 and TXNIP. Oncogene 2019;38(11):1892-1904. doi:10.1038/s41388-018-0534-3.

[4] Shi L, Middleton J, Jeon YJ, Magee P, Veneziano D, Laganà A, et al. KRAS induces lung tumorigenesis through microRNAs modulation. Cell Death Dis 2018;9(2):219. doi:10.1038/s41419-017-0243-9.

[5] Wang $\mathrm{Y}$, Zhang S, Bao H, Mu S, Zhang B, Ma H, et al. MicroRNA-365 promotes lung carcinogenesis by downregulating the USP33/SLIT2/ ROBO1 signalling pathway. Cancer Cell Int 2018;18:64. doi:10.1186/ s12935-018-0563-6.

[6] Jeon SH, Yoo JK, Kim CM, Lim ES, Lee SJ, Lee JM, et al. The novel hsamiR-12528 regulates tumourigenesis and metastasis through hypophosphorylation of AKT cascade by targeting IGF-1R in human lung cancer. Cell Death Dis 2018;9(5):493. doi:10.1038/s41419-018-05358.

[7] Fujii T, Shimada K, Nakai T, Ohbayashi C. MicroRNAs in Smoking-Related Carcinogenesis: Biomarkers, Functions, and Therapy. J Clin Med 2018;7(5):98. doi:10.3390/jcm7050098.

[8] Zhang L, Wang H, Wang C. Persistence of smoking induced nonsmall cell lung carcinogenesis by decreasing ERBB pathway-related microRNA expression. Thorac Cancer 2019;10(4):890-897. doi:10.1111/1759-7714.13020.

[9] Du X, Qi F, Lu S, Li Y, Han W. Nicotine upregulates FGFR3 and RB1 expression and promotes non-small cell lung cancer cell proliferation and epithelial-to-mesenchymal transition via downregulation of miR-99b and miR-192. Biomed Pharmacother 2018;101:656-662. doi:10.1016/j.biopha.2018.02.113.

[10] Lu L, Xu H, Yang P, Xue J, Chen C, Sun Q, et al. Involvement of HIF-1alpha-regulated miR-21, acting via the Akt/NF-kappaB pathway, in malignant transformation of HBE cells induced by cigarette smoke extract. Toxicol Lett 2018;289:14-21. doi:10.1016/j.toxlet.2018.02.027.

[11] LuJ,ZhongY,ChenJ, LinX, LinZ, Wang N, etal. RadiationEnhancestheEpithelial-Mesenchymal Transition of A549 Cells via miR3591-5p/USP33/ PPM1A. Cell Physiol Biochem 2018;50(2):721-733. doi:10.1159/ 000494238.

[12] Zhou B, Wang D, Sun G, Mei F, Cui Y, Xu H. Effect of miR-21 on Apoptosis in Lung Cancer Cell Through Inhibiting the PI3K/Akt/ NF-kB Signaling Pathway in Vitro and in Vivo. Cell Physiol Biochem 2018;46(3):999-1008. doi:10.1159/000488831.

[13] Baumgartner U, Berger F, Hashemi Gheinani A, Burgener SS, Monastyrskaya K, Vassella E. miR-19b enhances proliferation and apoptosis resistance via the EGFR signaling pathway by targeting PP2A and BIM in non-small cell lung cancer. Mol Cancer 2018;17(1):44. doi:10.1186/ s12943-018-0781-5.

[14] Dong YZ, Hu T. Effects of miR-143 overexpression on proliferation, apoptosis, EGFR and downstream signaling pathways in PC9/GR cell line. Eur Rev Med Pharmacol Sci 2018;22(6):1709-1716. doi:10.26355/ eurrev_201803_14584.

[15] Meng X L, Li Z, Zhou S, Xiao S, Yu P. miR-194 suppresses high glucoseinduced non-small cell lung cancer cell progression by targeting NFAT5. Thorac Cancer 2019;10(5):1051-1059. doi:10.1111/17597714.13038

[16] Zhou Y, Li S, Li J, Wang D, Li Q. Effect of microRNA-135a on Cell Proliferation, Migration, Invasion, Apoptosis and Tumor Angiogenesis Through the IGF-1/PI3K/Akt Signaling Pathway in Non-Small Cell Lung Cancer. Cell Physiol Biochem 2017;42(4):1431-1446. doi:10.1159/ 000479207.

[17] Huang RS, Zheng YL, Li C, Ding C, Xu C, Zhao J. MicroRNA-485-5p suppresses growth and metastasis in non-small cell lung cancer cells by targeting IGF2BP2. Life Sci 2018;199:104-111. doi:10.1016/j. Ifs.2018.03.005
[18] Yang CL, Zheng XL, Ye K, Ge H, Sun YN, Lu YF, et al. MicroRNA-183 Acts as a Tumor Suppressor in Human Non-Small Cell Lung Cancer by Down-Regulating MTA1. Cell Physiol Biochem 2018;46(1):93-106. doi:10.1159/000488412.

[19] Liu J, Bian T, Feng J, Qian L, Zhang J, Jiang D, et al. miR-335 inhibited cell proliferation of lung cancer cells by target Tra2 $\beta$. Cancer Sci 2018;109(2):289-296. doi:10.1111/cas.13452.

[20] Zhao L, Ni X, Zhao L, Zhang Y, Jin D, Yin W, et al. MiroRNA-188 Acts as Tumor Suppressor in Non-Small-Cell Lung Cancer by Targeting MAP3K3. Mol Pharm 2018;15(4):1682-1689. doi:10.1021/acs. molpharmaceut.8b00071.

[21] Ruan L, Chen J, Ruan L, Yang T, Wang P. MicroRNA-186 suppresses lung cancer progression by targeting SIRT6. Cancer Biomark 2018;21(2):415-423. doi:10.3233/cbm-170650.

[22] Yang W, Bai J, Liu D, Wang S, Zhao N, Che R, et al. MiR-93-5p up-regulation is involved in non-small cell lung cancer cells proliferation and migration and poor prognosis. Gene 2018;647:13-20. doi:10.1016/j. gene.2018.01.024

[23] Xu G, Zhang Z, Zhang L, Chen Y, Li N, Lv Y, et al. miR-4326 promotes lung cancer cell proliferation through targeting tumor suppressor APC2. Mol Cell Biochem 2018;443(1-2):151-157. doi:10.1007/ s11010-017-3219-2.

[24] Ren H, Zhang Y, Zhu H. MiR-339 depresses cell proliferation via directly targeting S-phase kinase-associated protein $2 \mathrm{mRNA}$ in lung cancer. Thorac Cancer 2018;9(3):408-414. doi:10.1111/1759-7714.12597.

[25] Jin K, Zhao W, Xie X, Pan Y, Wang K, Zhang H. MiR-520b restrains cell growth by targeting HDAC4 in lung cancer. Thorac Cancer 2018;9(10):1249-1254. doi:10.1111/1759-7714.12825.

[26] Pan J, Jiang F, Zhou J, Wu D, Sheng Z, Li M. HSP90: A Novel Target Gene of miRNA-628-3p in A549 Cells. Biomed Res Int 2018;2018:4149707. doi:10.1155/2018/4149707.

[27] Zhao W, Hu JX, Hao RM, Zhang Q, Guo JQ, Li YJ, et al. Induction of microRNA-let-7a inhibits lung adenocarcinoma cell growth by regulating cyclin D1. Oncol Rep 2018;40(4):1843-1854. doi:10.3892/ or.2018.6593.

[28] Tang H, Lv W, Sun W, Bi Q, Hao Y. miR-505 inhibits cell growth and EMT by targeting MAP3K3 through the AKT-NFKB pathway in NSCLC cells. Int J Mol Med 2019;43(3):1203-1216. doi:10.3892/ijmm.2018.4041.

[29] Li G, Li M, Hu J, Lei R, Xiong H, Ji H, et al. The microRNA-182-PDK4 axis regulates lung tumorigenesis by modulating pyruvate dehydrogenase and lipogenesis. Oncogene 2017;36(7):989-998. doi:10.1038/ onc.2016.265.

[30] Subramaniam S, Jeet V, Clements JA, Gunter JH, Batra J. Emergence of MicroRNAs as Key Players in Cancer Cell Metabolism. Clin Chem 2019;65(9):1090-1101. doi:10.1373/clinchem.2018.299651.

[31] Vander Heiden MG, Cantley LC, Thompson CB. Understanding the Warburg effect: the metabolic requirements of cell proliferation. Science 2009;324(5930):1029-1033. doi:10.1126/science.1160809.

[32] Lei W, Kang W, Nan Y, Lei Z, Zhongdong L, Demin L, et al. The Downregulation of miR-200c Promotes Lactate Dehydrogenase A Expression and Non-Small Cell Lung Cancer Progression. Oncol Res 2018:26(7):1015-1022. doi:10.3727/096504018×15151486241153.

[33] Zhai S, Zhao L, Lin T, Wang W. Downregulation of miR-33b promotes non-small cell lung cancer cell growth through reprogramming glucose metabolism miR-33b regulates non-small cell lung cancer cell growth. J Cell Biochem 2019;120(4):6651-6660. doi:10.1002/ jcb.27961.

[34] Zhang K, Zhang M, Jiang H, Liu F, Liu H, Li Y. Down-regulation of miR214 inhibits proliferation and glycolysis in non-small-cell lung cancer cells via down-regulating the expression of hexokinase 2 and pyruvate kinase isozyme M2. Biomed Pharmacother 2018;105:545-552. doi:10.1016/j.biopha.2018.06.009.

[35] Wu S, Zhang G, Li P, Chen S, Zhang F, Li J, et al. miR-198 targets SHMT1 to inhibit cell proliferation and enhance cell apoptosis in lung adenocarcinoma. Tumour Biol 2016;37(4):5193-5202. doi:10.1007/ s13277-015-4369-z.

[36] Lee SS, Cheah YK. The Interplay between MicroRNAs and Cellular Components of Tumour Microenvironment (TME) on Non-Small-Cell Lung Cancer (NSCLC) Progression. J Immunol Res 2019;2019:3046379. doi:10.1155/2019/3046379.

[37] Wang Y, Wang L, Chen C, Chu X. New insights into the regulatory role 
of microRNA in tumor angiogenesis and clinical implications. Mol Cancer 2018;17(1):22. doi:10.1186/s12943-018-0766-4.

[38] Dimitrova N, Gocheva V, Bhutkar A, Resnick R, Jong RM, Miller KM, et al. Stromal Expression of miR-143/145 Promotes Neoangiogenesis in Lung Cancer Development. Cancer Discov 2016;6(2):188-201. doi:10.1158/2159-8290.Cd-15-0854.

[39] Hsu YL, Hung JY, Chang WA, Lin YS, Pan YC, Tsai PH, et al. Hypoxic lung cancer-secreted exosomal miR-23a increased angiogenesis and vascular permeability by targeting prolyl hydroxylase and tight junction protein ZO-1. Oncogene 2017;36(34):4929-4942. doi:10.1038/ onc.2017.105

[40] Korde A, Jin L, Zhang JG, Ramaswamy A, Hu B, Kolahian S, et al. Lung Endothelial MicroRNA-1 Regulates Tumor Growth and Angiogenesis. Am J Respir Crit Care Med 2017;196(11):1443-1455. doi:10.1164/ rccm.201610-21570C.

[41] Liu X, Gao X, Zhang W, Zhu T, Bi W, Zhang Y. MicroRNA-204 deregulation in lung adenocarcinoma controls the biological behaviors of endothelial cells potentially by modulating Janus kinase 2-signal transducer and activator of transcription 3 pathway. IUBMB Life 2018;70(1):81-91. doi:10.1002/iub.1706.

[42] Jin X, Yu Y, Zou Q, Wang M, Cui Y, Xie J, et al. MicroRNA-105 promotes epithelial-mesenchymal transition of nonsmall lung cancer cells through upregulating Mcl-1. J Cell Biochem 2019;120(4):5880-5888. doi:10.1002/jcb.27873.

[43] Yang Y, Sun Y, Wu Y, Tang D, Ding X, Xu W, et al. Downregulation of miR-3127-5p promotes epithelial-mesenchymal transition via FZD4 regulation of $\mathrm{Wnt} / \beta$-catenin signaling in non-small-cell lung cancer. Mol Carcinog 2018;57(7):842-853. doi:10.1002/mc.22805.

[44] Li Y, Zhang H, Li Y, Zhao C, Fan Y, Liu J, et al. MiR-182 inhibits the epithelial to mesenchymal transition and metastasis of lung cancer cells by targeting the Met gene. Mol Carcinog 2018;57(1):125-136. doi:10.1002/mc.22741.

[45] Yang F, Wei K, Qin Z, Liu W, Shao C, Wang C, et al. MiR-598 Suppresses Invasion and Migration by Negative Regulation of Derlin-1 and Epithelial-Mesenchymal Transition in Non-Small Cell Lung Cancer. Cell Physiol Biochem 2018;47(1):245-256. doi:10.1159/000489803.

[46] Yin Q, Han Y, Zhu D, Li Z, Shan S, Jin W, et al. miR-145 and miR-497 suppress TGF- $\beta$-induced epithelial-mesenchymal transition of non-small cell lung cancer by targeting MTDH. Cancer Cell Int 2018;18:105. doi:10.1186/s12935-018-0601-4.

[47] Hsu YL, Hung JY, Chang WA, Jian SF, Lin YS, Pan YC, et al. Hypoxic Lung-Cancer-Derived Extracellular Vesicle MicroRNA-103a Increases the Oncogenic Effects of Macrophages by Targeting PTEN. Mol Ther 2018;26(2):568-581. doi:10.1016/j.ymthe.2017.11.016.

[48] Xu X, Cao L, Zhang Y, Lian H, Sun Z, Cui Y. MicroRNA-1246 inhibits cell invasion and epithelial mesenchymal transition process by targeting CXCR4 in lung cancer cells. Cancer Biomark 2018;21(2):251-260. doi:10.3233/cbm-170317.

[49] Li D, He C, Wang J, Wang Y, Bu J, Kong X, et al. MicroRNA-138 Inhibits Cell Growth, Invasion, and EMT of Non-Small Cell Lung Cancer via SOX4/p53 Feedback Loop. Oncol Res 2018;26(3):385-400. doi:10.37 27/096504017x14973124850905.

[50] Zhang X, Sai B, Wang F, Wang L, Wang Y, Zheng L, et al. Hypoxic BMSCderived exosomal miRNAs promote metastasis of lung cancer cells via STAT3-induced EMT. Mol Cancer 2019;18(1):40. doi:10.1186/ s12943-019-0959-5.

[51] Li Q, Ran P, Zhang X, Guo X, Yuan Y, Dong T, et al. Downregulation of N-Acetylglucosaminyltransferase GCNT3 by miR-302b-3p Decreases Non-Small Cell Lung Cancer (NSCLC) Cell Proliferation, Migration and Invasion. Cell Physiol Biochem 2018;50(3):987-1004. doi:10.1159/000494482.

[52] Song Q, Ji Q, Xiao J, Li F, Wang L, Chen Y, et al. miR-409 Inhibits Human Non-Small-Cell Lung Cancer Progression by Directly Targeting SPIN1. Mol Ther Nucleic Acids 2018;13:154-163. doi:10.1016/j. omtn.2018.08.020.

[53] Lv X, Li CY, Han P, Xu XY. MicroRNA-520a-3p inhibits cell growth and metastasis of non-small cell lung cancer through PI3K/AKT/mTOR signaling pathway. Eur Rev Med Pharmacol Sci 2018;22(8):23212327. doi:10.26355/eurrev 20180414822

[54] Xie S, Liu G, Huang J, Hu HB, Jiang W. miR-210 promotes lung adenocarcinoma proliferation, migration, and invasion by targeting lysyl oxidase-like 4. J Cell Physiol 2019;234(8):14050-14057. doi:10.1002/ jcp. 28093.

[55] Xue X, Fei X, Hou W, Zhang Y, Liu L, Hu R. miR-342-3p suppresses cell proliferation and migration by targeting AGR2 in non-small cell lung cancer. Cancer Lett 2018;412:170-178. doi:10.1016/j.canlet.2017.10.024.

[56] Pan L, Tang Z, Pan L, Tang R. MicroRNA-3666 inhibits lung cancer cell proliferation, migration, and invasiveness by targeting BPTF. Biochem Cell Biol 2019;97(4):415-422. doi:10.1139/bcb-2018-0301.

[57] Sun X, Cui S, Fu X, Liu C, Wang Z, Liu Y. MicroRNA-146-5p promotes proliferation, migration and invasion in lung cancer cells by targeting claudin-12. Cancer Biomark 2019;25(1):89-99. doi:10.3233/cbm182374.

[58] Kang M, Li Y, Zhao Y, He S, Shi J. miR-33a inhibits cell proliferation and invasion by targeting CAND1 in lung cancer. Clin Transl Oncol 2018;20(4):457-466. doi:10.1007/s12094-017-1730-2.

[59] Han X, Tang Y, Dai Y, Hu S, Zhou J, Liu X, et al. MiR-889 promotes cell growth in human non-small cell lung cancer by regulating KLF9. Gene 2019;699:94-101. doi:10.1016/j.gene.2019.02.077.

[60] Yang D, Wang JJ, Li JS, Xu QY. miR-103 Functions as a Tumor Suppressor by Directly Targeting Programmed Cell Death 10 in NSCLC. Oncol Res 2018;26(4):519-528. doi:10.3727/096504017x15000757094686.

[61] Dou L, Han K, Xiao M, Lv F. miR-223-5p Suppresses Tumor Growth and Metastasis in Non-Small Cell Lung Cancer by Targeting E2F8. Oncol Res 2019;27(2):261-268. doi:10.3727/096504018x15219188894056.

[62] Wu D, Liu J, Chen J, He H, Ma H, Lv X. miR-449a Suppresses Tumor Growth, Migration, and Invasion in Non-Small Cell Lung Cancer by Targeting a HMGB1-Mediated NF-кB Signaling Pathway. Oncol Res 2019;27(2):227-235. doi:10.3727/096504018x15213089759999.

[63] Wang L, Qu J, Zhou L, Liao F, Wang J. MicroRNA-373 Inhibits Cell Proliferation and Invasion via Targeting BRF2 in Human Non-small Cell Lung Cancer A549 Cell Line. Cancer Res Treat 2018;50(3):936-949. doi:10.4143/crt.2017.302.

[64] Chen X, Du J, Jiang R, Li L. MicroRNA-214 inhibits the proliferation and invasion of lung carcinoma cells by targeting JAK1. Am J Trans Res 2018;10(4):1164-1171.

[65] Han L, Chen W, Xia Y, Song Y, Zhao Z, Cheng H, et al. MiR-101 inhibits the proliferation and metastasis of lung cancer by targeting zinc finger E-box binding homeobox 1. Am J Transl Res 2018;10(4):11721183.

[66] Li P, Wang Q, Wang H. MicroRNA-204 inhibits the proliferation, migration and invasion of human lung cancer cells by targeting PCNA-1 and inhibits tumor growth in vivo. Int J Mol Med 2019;43(3):11491156. doi:10.3892/ijmm.2018.4044.

[67] Zhou GH, Lu YY, Xie JL, Gao ZK, Wu XB, Yao WS, et al. Overexpression of miR-758 inhibited proliferation, migration, invasion, and promoted apoptosis of non-small cell lung cancer cells by negatively regulating HMGB. Biosci Rep 2019;39(1):BSR20180855. doi:10.1042/ bsr20180855.

[68] Li G, Wang K, Wang J, Qin S, Sun X, Ren H. miR-497-5p inhibits tumor cell growth and invasion by targeting SOX5 in non-small-cell lung cancer. J Cell Biochem 2019;120(6):10587-10595. doi:10.1002/ jcb. 28345.

[69] Yang F, Xiong H, Duan L, Li Q, Li X, Zhou Y. MiR-1246 Promotes Metastasis and Invasion of A549 cells by Targeting GSK-3ß-Mediated Wnt/ $\beta$-Catenin Pathway. Cancer Res Treat 2019;51(4):1420-1429. doi:10.4143/crt.2018.638.

[70] Luo D, Zhang Z, Zhang Z, Li JY, Cui J, Shi WP, et al. Aberrant Expression of miR-362 Promotes Lung Cancer Metastasis through Downregulation of Sema3A. J Immunol Res 2018;2018:1687097. doi:10.1155/2018/1687097.

[71] Frixa T, Sacconi A, Cioce M, Roscilli G, Ferrara FF, Aurisicchio L, et al. MicroRNA-128-3p-mediated depletion of Drosha promotes lung cancer cell migration. Carcinogenesis 2018;39(2):293-304. doi:10.1093/ carcin/bgx134.

[72] Zhao W, Sun Q, Yu Z, Mao S, Jin Y, Li J, et al. MiR-320a-3p/ELF3 axis regulates cell metastasis and invasion in non-small cell lung cancer via PI3K/Akt pathway. Gene 2018;670:31-37. doi:10.1016/j.gene.2018. 05.100 .

[73] Wang J, Lu Y, Ding H, Gu T, Gong C, Sun J, et al. The miR-875-5p inhibits SATB2 to promote the invasion of lung cancer cells. Gene 
2018;644:13-19. doi:10.1016/j.gene.2017.11.066.

[74] SongQ,LiuB,LiX,ZhangQ,CaoL,XuM, etal.MiR-26a-5ppotentiatesmetastasis of humanlung cancercells byregulating ITG $\beta 8$-JAK2/STAT3axis. Biochem Biophys Res Commun 2018;501(2):494-500. doi:10.1016/ j.bbrc.2018.05.020.

[75] Xing A, Pan L, Gao J. p100 functions as a metastasis activator and is targeted by tumor suppressing microRNA-320a in lung cancer. Thorac Cancer 2018;9(1):152-158. doi:10.1111/1759-7714.12564.

[76] Chen GM, Zheng AJ, Cai J, Han P, Ji HB, Wang LL. microRNA-145-3p inhibits non-small cell lung cancer cell migration and invasion by targeting PDK1 via the mTOR signaling pathway. J Cell Biochem 2018;119(1): 885-895. doi:10.1002/jcb.26252.

[77] Chen W, Huang Y, Zhang S, Zheng X, Xie S, Mao J, et al. MicroRNA-212 suppresses nonsmall lung cancer invasion and migration by regulating ubiquitin-specific protease-9. J Cell Biochem 2019;120(4):64826489. doi:10.1002/jcb.27939.

[78] Yan L, Ma J, Zhu Y, Zan J, Wang Z, Ling L, et al. miR-24-3p promotes cell migration and proliferation in lung cancer by targeting SOX7. J Cell Biochem 2018;119(5):3989-3998. doi:10.1002/jcb.26553.

[79] Jiang K, Shen M, Chen Y, Xu W. miR-150 promotes the proliferation and migration of non-small cell lung cancer cells by regulating the SIRT2/JMJD2A signaling pathway. Oncol Rep 2018;40(2):943-951. doi:10.3892/or.2018.6487.

[80] Zhang X, Wang Q, Zhang S. MicroRNAs in sputum specimen as noninvasive biomarkers for the diagnosis of nonsmall cell lung cancer: An updated meta-analysis. Medicine (Baltimore) 2019;98(6):e14337. doi:10.1097/md.0000000000014337.

[81] Leng $Q$, Wang Y, Jiang F. A Direct Plasma miRNA Assay for Early Detection and Histological Classification of Lung Cancer. Transl Oncol 2018;11(4):883-889. doi:10.1016/j.tranon.2018.05.001.

[82] Zhou GH, Yang WH, Sun B. Clinical impact of serum miR-661 in diagnosis and prognosis of non-small cell lung cancer. Eur Rev Med Pharmacol Sci 2017;21(24):5696-5701. doi:10.26355/eurrev_201712 14015.

[83] Wang SY, Li Y, Jiang YS, Li RZ. Investigation of serum miR-411 as a diagnosis and prognosis biomarker for non-small cell lung cancer. Eur Rev Med Pharmacol Sci 2017;21(18):4092-4097.

[84] Lu J, Zhan Y, Feng J, Luo J, Fan S. MicroRNAs associated with therapy of non-small cell lung cancer. Int J Biol Sci 2018;14(4):390-397. doi:10.7150/ijbs.22243.

[85] Cai J, Fang L, Huang Y, Li R, Xu X, Hu Z, et al. Publisher Correction: Simultaneous overactivation of Wnt/ $\beta$-catenin and TGF $\beta$ signalling by miR$128-3 p$ confers chemoresistance-associated metastasis in NSCLC. Nat Commun 2018;9:16196. doi:10.1038/ncomms16196.

[86] Wu L, Pu X, Wang Q, Cao J, Xu F, Xu LI, et al. miR-96 induces cisplatin chemoresistance in non-small cell lung cancer cells by downregulating SAMD9. Oncol Lett 2016;11(2):945-952. doi:10.3892/ol.2015. 4000.

[87] Zhang Q, Zhang B, Sun L, Yan Q, Zhang Y, Zhang Z, et al. MicroRNA-130b targets PTEN to induce resistance to cisplatin in lung cancer cells by activating Wnt/ $\beta$-catenin pathway. Cell Biochem Funct 2018;36(4):194 -202. doi:10.1002/cbf.3331.

[88] Deng H, Qianqian G, Ting J, Aimin Y. miR-539 enhances chemosensitivity to cisplatin in non-small cell lung cancer by targeting DCLK1. Biomed Pharmacother 2018;106:1072-1081. doi:10.1016/j.biopha. 2018.07.024.

[89] Liu HN, Qie P, Yang G, Song YB. miR-181b inhibits chemoresistance in cisplatin-resistant $\mathrm{H} 446$ small cell lung cancer cells by targeting Bcl-2. Arch Med Sci 2018;14(4):745-751. doi:10.5114/aoms.2018.73131.

[90] Sun W, Ping W, Tian Y, Zou W, Liu J, Zu Y. miR-202 Enhances the AntiTumor Effect of Cisplatin on Non-Small Cell Lung Cancer by Targeting the Ras/MAPK Pathway. Cell Physiol Biochem 2018;51(5):21602171. doi:10.1159/000495835.

[91] Wang N, Zhu C, Xu Y, Qian W, Zheng M. Negative Regulation of PTEN by MicroRNA-221 and Its Association with Drug Resistance and Cellular Senescence in Lung Cancer Cells. Biomed Res Int 2018;2018: 7908950. doi:10.1155/2018/7908950.

[92] Van Roosbroeck K, Fanini F, Setoyama T, Ivan C, Rodriguez-Aguayo C, Fuentes-Mattei E, et al. Combining Anti-Mir-155 with Chemotherapy for the Treatment of Lung Cancers. Clin Cancer Res 2017;23(11): 2891-2904. doi:10.1158/1078-0432.Ccr-16-1025.
[93] Lin C, Xie L, Lu Y, Hu Z, Chang J. miR-133b reverses cisplatin resistance by targeting GSTP1 in cisplatin-resistant lung cancer cells. Int J Mol Med 2018;41(4):2050-2058. doi:10.3892/ijmm.2018.3382.

[94] Yu S, Qin X, Chen T, Zhou L, Xu X, Feng J. MicroRNA-106b-5p regulates cisplatin chemosensitivity by targeting polycystic kidney disease-2 in non-small-cell lung cancer. Anticancer Drugs 2017;28(8):852-860. doi:10.1097/cad.0000000000000524.

[95] Yin Y, Liu H, Xu J, Shi D, Zhai L, Liu B, et al. miR-144-3p regulates the resistance of lung cancer to cisplatin by targeting Nrf2. Oncol Rep 2018;40(6):3479-3488. doi:10.3892/or.2018.6772.

[96] Lin Z, Pan J, Chen L, Wang X, Chen Y. MiR-140 Resensitizes CisplatinResistant NSCLC Cells to Cisplatin Treatment Through the SIRT1/ROS/ JNK Pathway. Onco Targets Ther 2020;13:8149-8160. doi:10.2147/ ott.S261799.

[97] Ba Z, Zhou Y, Yang Z, Xu J, Zhang X. miR-324-5p upregulation potentiates resistance to cisplatin by targeting FBXO11 signalling in non-small cell lung cancer cells. J Biochem 2019;166(6):517-527. doi:10.1093/ $\mathrm{jb} / \mathrm{mvz066}$.

[98] Babar IA, Czochor J, Steinmetz A, Weidhaas JB, Glazer PM, Slack FJ. Inhibition of hypoxia-induced miR-155 radiosensitizes hypoxic lung cancer cells. Cancer Biol Ther 2011;12(10):908-914. doi:10.4161/cbt.12.10. 17681.

[99] Shin S, Cha HJ, Lee EM, Lee SJ, Seo SK, Jin HO, et al. Alteration of miRNA profiles by ionizing radiation in A549 human non-small cell lung cancer cells. Int J Oncol 2009;35(1):81-86.

[100] Shen Z, Wu X, Wang Z, Li B, Zhu X. Effect of miR-18a overexpression on the radiosensitivity of non-small cell lung cancer. Int J Clin Exp Pathol 2015;8(1):643-648.

[101] Guo Y, Jiang Y, Sang M, Xu C. RETRACTED: Down-regulation of miR373 increases the radiosensitivity of lung cancer cells by targeting TIMP2. Int J Biochem Cell Biol 2018;99:203-210. doi:10.1016/j.biocel.2018.04.014.

[102] Zhu L, Xue F, Xu X, Xu J, Hu S, Liu S, et al. MicroRNA-198 inhibition of HGF/c-MET signaling pathway overcomes resistance to radiotherapy and induces apoptosis in human non-small-cell lung cancer. J Cell Biochem 2018;119(9):7873-7886. doi:10.1002/jcb.27204.

[103] Chen X, Wu L, Li D, Xu Y, Zhang L, Niu K, et al. Radiosensitizing effects of miR-18a-5p on lung cancer stem-like cells via downregulating both ATM and HIF-1 $\alpha$. Cancer Med 2018;7(8):3834-3847. doi:10.1002/ cam4.1527.

[104] Jiang S, Wang R, Yan H, Jin L, Dou X, Chen D. MicroRNA-21 modulates radiation resistance through upregulation of hypoxia-inducible factor-1 $\alpha$-promoted glycolysis in non-small cell lung cancer cells. Mol Med Rep 2016;13(5):4101-4107. doi:10.3892/mmr.2016.5010.

[105] Du M, Wang J, Chen H, Wang S, Chen L, Xu Y, et al. MicroRNA-200a suppresses migration and invasion and enhances the radiosensitivity of NSCLC cells by inhibiting the HGF/c-Met signaling pathway. Oncol Rep 2019;41(3):1497-1508. doi:10.3892/or.2018.6925.

[106] Li Y, Han W, Ni TT, Lu L, Huang M, Zhang Y, et al. Knockdown of microRNA-1323 restores sensitivity to radiation by suppression of PRKDC activity in radiation-resistant lung cancer cells. Oncol Rep 2015;33(6):2821-2828. doi:10.3892/or.2015.3884.

[107] Song L, Peng L, Hua S, Li X, Ma L, Jie J, et al. miR-144-5p Enhances the Radiosensitivity of Non-Small-Cell Lung Cancer Cells via Targeting ATF2. Biomed Res Int 2018;2018:5109497. doi:10.1155/2018/510 9497.

[108] Yin H, Ma J, Chen L, Piao S, Zhang Y, Zhang S, et al. MiR-99a Enhances the Radiation Sensitivity of Non-Small Cell Lung Cancer by Targeting mTOR. Cell Physiol Biochem 2018;46(2):471-481. doi:10.1159/ 000488615.

[109] Yue J, Lv D, Wang C, Li L, Zhao Q, Chen H, et al. Epigenetic silencing of miR-483-3p promotes acquired gefitinib resistance and EMT in EGFR-mutant NSCLC by targeting integrin $\beta 3$. Oncogene 2018;37(31): 4300-4312. doi:10.1038/s41388-018-0276-2.

[110] Zhang T, Wang N. miR-135a Confers Resistance to Gefitinib in NonSmall Cell Lung Cancer Cells by Upregulation of RAC1. Oncol Res 2018;26(8):1191-1200. doi:10.3727/096504018x15166204902353.

[111] Ping W, Gao Y, Fan X, Li W, Deng Y, Fu X. MiR-181a contributes gefitinib resistance in non-small cell lung cancer cells by targeting GAS7. Biochem Biophys Res Commun 2018;495(4):2482-2489. doi:10.1016/ j.bbrc.2017.12.096. 
[112] Zhou G, Zhang F, Guo Y, Huang J, Xie Y, Yue S, et al. miR-200c enhances sensitivity of drug-resistant non-small cell lung cancer to gefitinib by suppression of PI3K/Akt signaling pathway and inhibites cell migration via targeting ZEB1. Biomed Pharmacother 2017;85:113-119. doi:10.1016/j.biopha.2016.11.100.

[113] Jin S, He J, Li J, Guo R, Shu Y, Liu P. MiR-873 inhibition enhances gefitinib resistance in non-small cell lung cancer cells by targeting gliomaassociated oncogene homolog 1. Thorac Cancer 2018;9(10):12621270. doi:10.1111/1759-7714.12830.

[114] Tang X, Jiang J, Zhu J, He N, Tan J. HOXA4-regulated miR-138 suppresses proliferation and gefitinib resistance in non-small cell lung cancer. Mol Genet Genomics 2019;294(1):85-93. doi:10.1007/s00438018-1489-3.

[115] Xie WB, Liang LH, Wu KG, Wang LX, He X, Song C, et al. MiR-140 Expression Regulates Cell Proliferation and Targets PD-L1 in NSCLC. Cell Physiol Biochem 2018;46(2):654-663. doi:10.1159/000488634.
[116] Ulivi P, Petracci E, Marisi G, Baglivo S, Chiari R, Billi M, et al. Prognostic Role of Circulating miRNAs in Early-Stage Non-Small Cell Lung Cancer. J Clin Med 2019;8(2):131. doi:10.3390/jcm8020131.

[117]Gao X, Zhao H, Diao C, Wang X, Xie Y, Liu Y, et al. miR-455-3p serves as prognostic factor and regulates the proliferation and migration of non-small cell lung cancer through targeting HOXB5. Biochem Biophys Res Commun 2018;495(1):1074-1080. doi:10.1016/j.bbrc.2017.11. 123.

[118] Li Y, Cui X, Li Y, Zhang T, Li S. Upregulated expression of miR-421 is associated with poor prognosis in non-small-cell lung cancer. Cancer Manag Res 2018;10:2627-2633. doi:10.2147/cmar.S167432.

[119] Zhang L, Lin J, Ye Y, Oba T, Gentile E, Lian J, et al. Serum MicroRNA-150 Predicts Prognosis for Early-Stage Non-Small Cell Lung Cancer and Promotes Tumor Cell Proliferation by Targeting Tumor Suppressor Gene SRCIN1. Clin Pharmacol Ther 2018;103(6):1061-1073. doi:10.1002/cpt.870. 